\title{
Macromodeling Based Variability Analysis of an Inverted Embedded Microstrip Line
}

\author{
Dries Vande Ginste \\ and Daniël De Zutter \\ Electromagnetics Group \\ Dept. of Information Technology \\ Ghent University \\ Gent, Belgium \\ Email:vdginste@intec.UGent.be
}

\author{
Dirk Deschrijver \\ and Tom Dhaene \\ Surrogate Modeling Lab \\ Dept. of Information Technology \\ Ghent University - IBBT \\ Gent, Belgium
}

\author{
Flavio Canavero \\ EMC Group \\ Dipartimento di Elettronica \\ Politecnico di Torino \\ Torino, Italy
}

\begin{abstract}
A multivariate macromodel of the per unit length parameters of an inverted embedded microstrip line is built starting from a limited number of highly accurate but computationally expensive electromagnetic simulations. Besides the frequency dependency, the macromodel also encompasses the influence of a geometrical parameter that determines the shape of the cross-section of the metallic interconnect. This allows to assess the influence of the etching process through a variability analysis of the interconnect's behavior in both frequency and time domain. The analysis is carried out via a robust Monte Carlo approach, which has been made computationally feasible thanks to the macromodeling step.
\end{abstract}

\section{INTRODUCTION}

Owing to further miniaturization and stringent specifications, on-chip interconnect design is becoming increasingly challenging. The manufacturing process induces random variations of geometrical and material parameters. These effects can no longer be neglected. Therefore, circuit designers should have access to tools that allow them to accurately assess the behavior of interconnects, and this preferably in such a way that design space explorations, sensitivity analysis, and variability analysis are feasible in an acceptable amount of time.

The inverted embedded microstrip (IEM) line is a typical on-chip interconnect structure that is gaining importance in circuit design [1]. Given its tiny dimensions, small geometrical variations, e.g. introduced by the etching process, can introduce a significant change of the behavior of the interconnect. This behavior should be known over a very broad frequency range, i.e. from DC up to deep skin-effect frequencies. Unfortunately, due to the presence of semiconductors, situated close to the actual metallic microstrip line, this behavior is hard to predict, let alone to be analytically obtained. This makes it hard to develop variability-aware modeling techniques. Hence, assessing the signal integrity behavior of such structures, taking process-induced geometrical parameter variations into account, is not straightforward.

In this contribution the effect of the etching on an IEM line is studied. Thereto, the following approach is adopted. The IEM line is characterized by means of its two-dimensional (2-D) per unit length (p.u.l.) resistance (R), inductance (L), conductance (G) and inductance (L) parameters. This frequency-dependent RLGC-behavior is determined using a very accurate, but slow, 2-D electromagnetic (EM) solver [2], [3]. Next, a multivariate macromodel [4] is built, taking the frequency-dependent behavior and also the effect of the etching into account. Thanks to an adaptive sampling technique (AFS) [5], the number of samples needed to construct the macromodel is limited, making this macromodeling step not too expensive. Once the macromodel is obtained, a brute-force Monte Carlo (MC) method [6] is implemented. The $\mathrm{MC}$ approach is in essence a sampling technique. It is wellknown that it is very robust, reliable and easy to implement, but that many samples are required before convergence is reached. Direct computation of these samples, using the 2-D EM solver, is not tractable. It will be shown that thanks to the macromodeling step, however, the MC approach becomes feasible, allowing an easy, robust, and efficient stochastic modeling of the IEM line.

\section{INVERTEd EMbedDEd MicrostriP (IEM) LiNE}

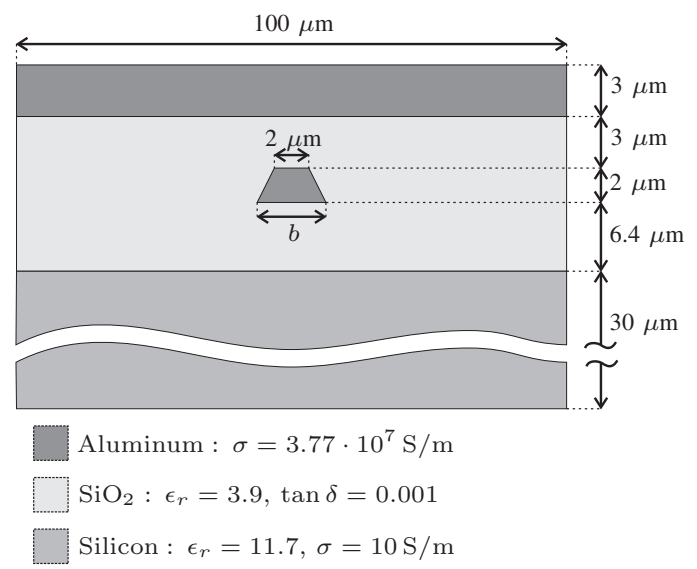

Fig. 1: Cross-section of the IEM line (not on scale).

The cross-section of the IEM line under consideration is shown in Fig. 1. This particular topology comprises a doped Silicon substrate with a thickness of $30 \mu \mathrm{m}$, a relative 
permittivity $\epsilon_{r}=11.7$, and a conductivity $\sigma=10 \mathrm{~S} / \mathrm{m}$ on which an insulator is placed. This insulator is $11.4 \mu \mathrm{m}$ thick $\mathrm{SiO}_{2}$ with a relative permittivity $\epsilon_{r}=3.9$ and a loss tangent $\tan \delta=0.001$. On top of the $\mathrm{SiO}_{2}$ the top-plate ground is found. This ground plate has a thickness of $3 \mu \mathrm{m}$ and is Aluminum with a conductivity $\sigma=3.77 \cdot 10^{7} \mathrm{~S} / \mathrm{m}$. The Aluminum interconnect is embedded in the $\mathrm{SiO}_{2}$ at a height of $6.4 \mu \mathrm{m}$ above the semiconductor. Due to the etching process, the cross-section of the line has a trapezoidal shape. The height of this trapezoid is fixed to $2 \mu \mathrm{m}$. The top side of the trapezoid is fixed to $2 \mu \mathrm{m}$ and the bottom side $b$ is variable.

\section{PARAMETERIZED MACROMODEL OF THE IEM LINE}

\section{A. 2-D EM RLGC modeling}

To very precisely determine the scalar, frequency dependent p.u.l. parameters $\mathrm{R}, \mathrm{L}, \mathrm{G}$, and $\mathrm{C}$ of the IEM line shown in Fig. 1, the technique presented in [2], [3] is used. This technique adopts a quasi-TM behavior of the fields. A careful definition of the circuit currents, taking semiconductors into account, leads to a complex capacitance and a complex inductance problem formulation. Making use of a discretized differential surface admittance operator, evolving from the Dirichletto-Neumann (DtN) boundary operator, the two problems are cast as boundary integral equations, which can be solved efficiently. All this leads to accurate, tabulated, p.u.l. parameters, from DC up to deep skin-effect frequencies, encompassing also slow-wave phenomena. Results of this computation are presented in Section III-C.

\section{B. Macromodeling}

To reduce the overall simulation cost, an Adaptive Frequency Sampling (AFS) algorithm is applied to compute the p.u.l. parameters only at a limited set of frequencies $f$ [5]. These data samples are subjected to Vector Fitting, which computes accurate frequency-dependent macromodels $F\left(f, b_{v}\right)$ for $V$ different values of parameter $b_{v}, v=1, \ldots, V$ [7]. Based on these univariate models, a bivariate model $F(f, b)$ is obtained by barycentric Lagrange interpolation [4]:

$$
F(f, b)=\left(\sum_{v=1}^{V} \frac{w_{v}}{b-b_{v}} F\left(f, b_{v}\right)\right) /\left(\sum_{v=1}^{V} \frac{w_{v}}{b-b_{v}}\right) .
$$

Since the data is smoothly varying in terms of the parameter $b$, the barycentric weights $w_{v}$ are chosen in such a way that the interpolation of the univariate models is polynomial [8].

\section{Numerical results}

For the construction of a macromodel that covers the frequency range from DC till $100 \mathrm{GHz}$ and for all values of the parameter $b \in[1,3] \mu \mathrm{m}$, we choose $V=4$ and thanks to AFS, only 116 tabulated samples are needed. The CPU time required to compute the p.u.l. parameters for each such sample with high precision on a standard laptop is $13.5 \mathrm{~s}$. Constructing the macromodel from these samples takes approximately $6 \mathrm{~s}$. So, the overall time to conceive the macromodel, was a bit less than half an hour. This can be considered as a setup cost for our further analysis purposes, described in Section IV.
The results of this modeling step are shown in Figs. 2 and 3, where the p.u.l. parameters are shown as a function of the frequency $f$ and as a function of the parameter $b$. The full lines $(-)$ are the result from the macromodel, the dots $(\bullet)$ indicate the initial samples used to build this model. The accuracy of the macromodel has been verified using validation samples (not indicated on the figures) for all four p.u.l. parameters and the maximum relative error is approximately $0.1 \%$. It is clear from the figures that, as expected, the four p.u.l. parameters have a strong frequency dependency and that the parameter $b$ has a significant influence. This influence will be further analyzed in the next section.

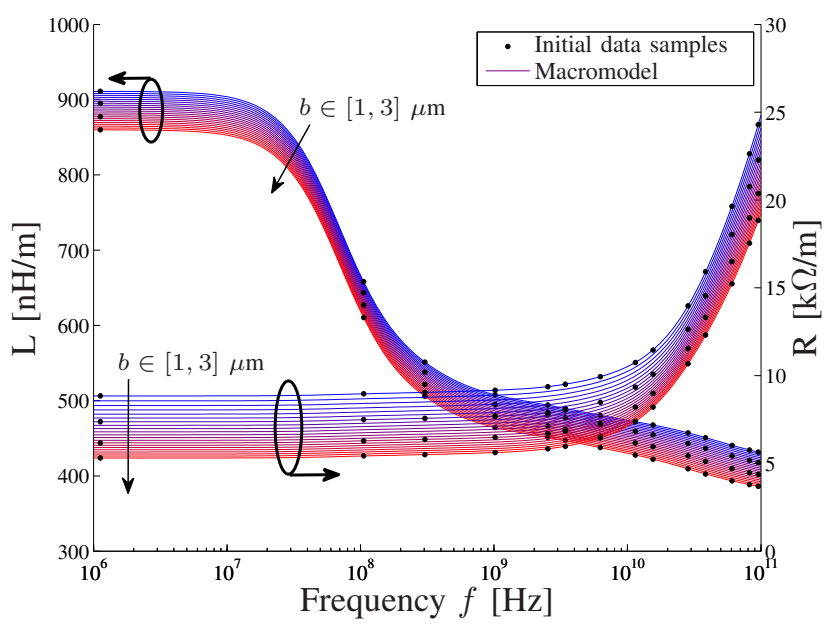

Fig. 2: $\mathrm{L}$ and $\mathrm{R}$ of the IEM line for 21 values of the parameter $b$, equidistantly spaced between $1 \mu \mathrm{m}$ and $3 \mu \mathrm{m}$.

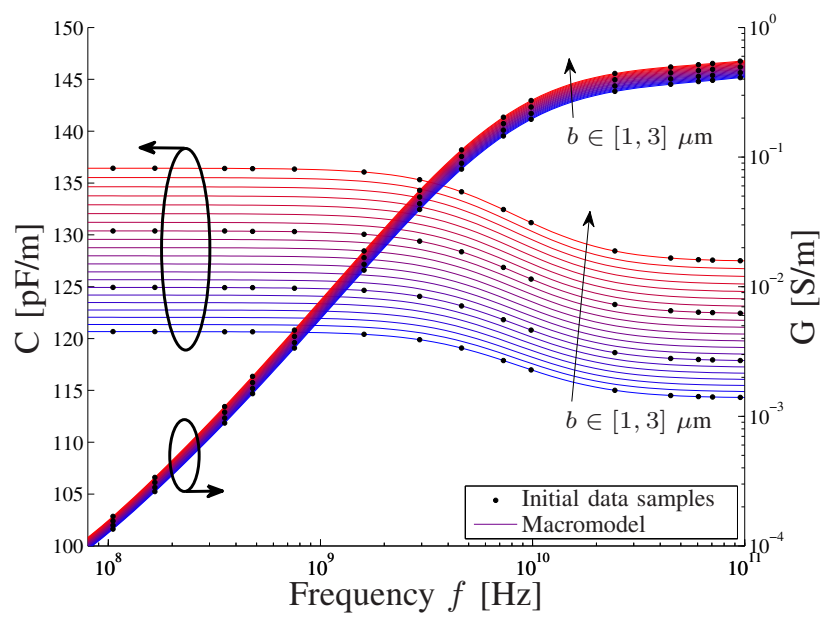

Fig. 3: $C$ and $G$ of the IEM line for 21 values of the parameter $b$, equidistantly spaced between $1 \mu \mathrm{m}$ and $3 \mu \mathrm{m}$.

\section{VARIABILITY ANALYSIS}

Computing many RLGC-samples for a wide range of frequencies $f$ and values of the parameter $b$, using the direct 
approach described in Section III-A, is not feasible due to the computational cost. Thanks to the macromodel, however, efficient design space explorations are now possible, as well as sensitivity analysis using a perturbation method, as well as optimizations. Here, a variability analysis of the influence of the etching process is performed by means of stochastic modeling, which is simply implemented adopting the wellknown, robust MC approach.

\section{A. Frequency domain analysis}

Consider a source-line-load configuration where the IEM line of Fig. 1 has a finite length of $1 \mathrm{~mm}$. The line is driven by a voltage source producing a sine wave with frequency $f$, amplitude $E(f)$, and with an internal impedance of $1 \Omega$. It is terminated by a load impedance $Z_{L}=\left(j 2 \pi f C_{L}+1 / R_{L}\right)^{-1}$, with $C_{L}=1 \mathrm{pF}$ and $R_{L}=1 \mathrm{k} \Omega$. To analyze the effect of the etching, an $\mathrm{MC}$ run is performed using 50000 samples of the parameter $b$, that is assumed to be a Gaussian random variable with mean $\mu_{b}=2 \mu \mathrm{m}$ and standard deviation $\sigma_{b}=0.2 \mu \mathrm{m}=$ $10 \% \mu_{b}$. A bode plot of the magnitude of the transfer function $H(f)=V_{L}(f) / E(f)$, with $V_{L}(f)$ the voltage at the load, is shown in Fig. 4. The gray lines correspond to 100 samples of the MC run, the full black line indicates the mean $\mu_{H}$ of the transfer function, computed using the 50000 samples, and the dashed lines show the $\pm 3 \sigma_{H}$ deviations from the mean $\mu_{H}$, where the standard deviation $\sigma_{H}$ is also calculated using the 50000 samples. In Fig. 5 the probability density function (PDF) and the cumulative distribution function (CDF) of $|H(f)|$ for $f=7 \mathrm{GHz}$ are shown, computed from the 50000 samples. This quantifies the influence of the variable parameter $b$, and hence the effect of the etching process, on the behavior of the interconnect. In Fig. 6 the relative error on the computed mean $\mu_{H}$ and standard deviation $\sigma_{H}$ of the magnitude of the transfer function $H(f)$ at $7 \mathrm{GHz}$ is shown as a function of $N$, i.e. the number of MC samples used. As expected, a $1 / \sqrt{N}$-convergence rate is obtained. It is also observed that using 50000 samples is clearly sufficient for our needs. The computation of the results presented in this section do not require a lot of CPU time. Computing the transfer function $H(f)$ for 250 frequency samples and for 50000 samples of $b$ only takes $4.8 \mathrm{~s}$, thanks to the macromodel.

\section{B. Time domain analysis}

Consider a similar source-line-load configuration where the IEM line of Fig. 1 again has a finite length of $1 \mathrm{~mm}$. Now the line is driven by a voltage source producing a ramped step from $0 \mathrm{~V}$ to $1 \mathrm{~V}$ with a rise time of $30 \mathrm{ps}$. This voltage source has an internal impedance of $1 \Omega$ and the line is terminated by a load impedance $Z_{L}=\left(j 2 \pi f C_{L}+1 / R_{L}\right)^{-1}$, with $C_{L}=1 \mathrm{pF}$ and $R_{L}=1 \mathrm{k} \Omega$. Again, an $\mathrm{MC}$ run is performed, using 50000 samples of the parameter $b$, assuming that it is a Gaussian random variable with mean $\mu_{b}=2 \mu \mathrm{m}$ and standard deviation $\sigma_{b}=0.2 \mu \mathrm{m}=10 \% \mu_{b}$. The voltage $V_{L}(t)$ at the load as a function of the time $t$ is shown in Fig. 7. The gray lines correspond to 100 samples of the MC run, the

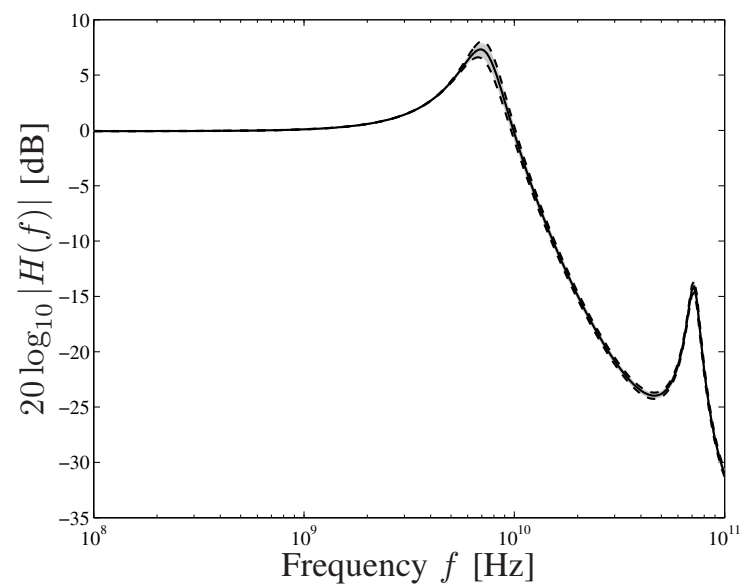

(a) Response as a function of frequency

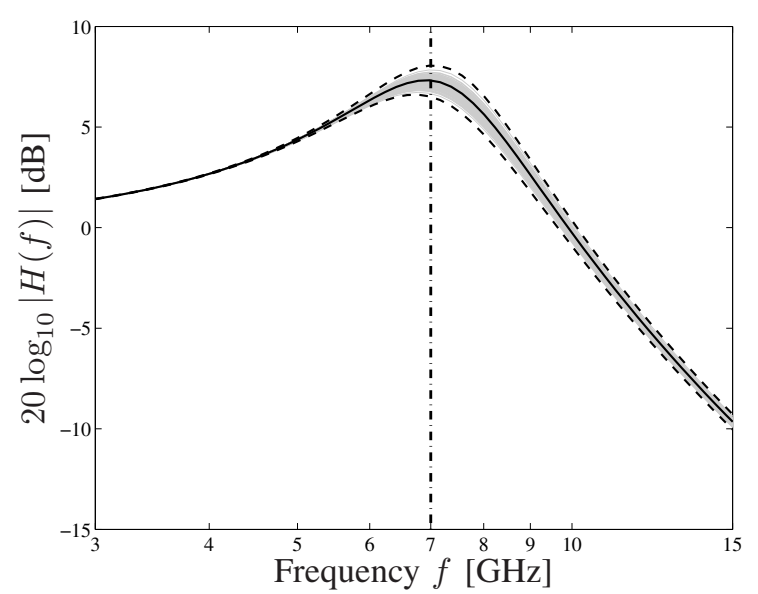

(b) Blow-up of Fig. 4(a) around $7 \mathrm{GHz}$, clearly illustrating the effect of the variable parameter $b$

Fig. 4: Bode plot of the magnitude of the transfer function $H(f)$ for the source-line-load example described in Section IV-A. Gray lines: MC samples (only 100 samples out of 50000 are shown); solid black line: mean $\mu_{H}$; dashed black lines: 3-sigma deviations $\mu_{H} \pm 3 \sigma_{H}$.

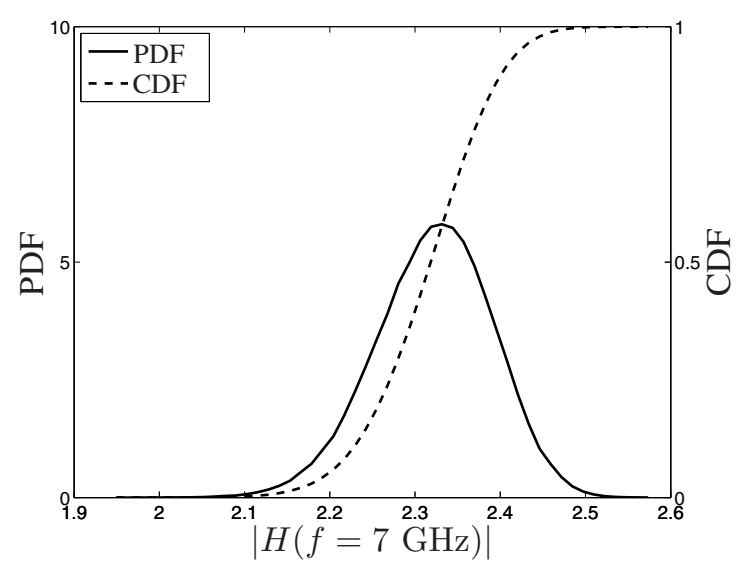

Fig. 5: PDF and CDF of the magnitude of the transfer function $H(f)$ at $7 \mathrm{GHz}$. 


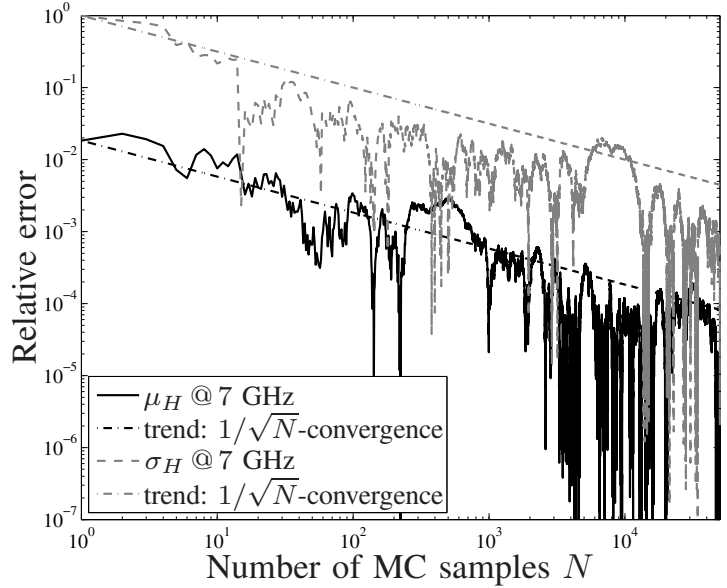

Fig. 6: Convergence of the MC simulation: relative error on the computed mean $\mu_{H}$ and standard deviation $\sigma_{H}$ of the magnitude of the transfer function $H(f=7 \mathrm{GHz})$, as a function of the number of MC samples used.

full black line indicates the mean $\mu_{V_{L}}$ of $V_{L}$ at each moment in time, computed using the 50000 samples, and the dashed lines show the $\pm 3 \sigma_{V_{L}}$ deviations from this mean $\mu_{V_{L}}$, where the standard deviation $\sigma_{V_{L}}$ is also calculated using the 50000 samples. In Fig. 8, the PDF and the CDF of the maximum overshoot, i.e. $\max _{t \geq 0}\left(V_{L}(t)\right)$, are shown, computed from the 50000 samples. This again clearly illustrates the influence of the variable parameter $b$, and hence, the small effect that the etching process has on the behavior of this interconnect structure.

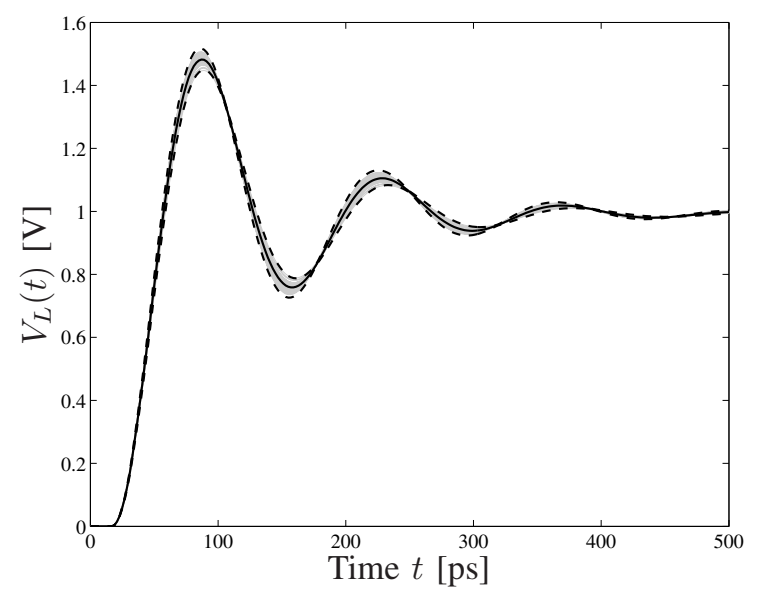

Fig. 7: Voltage $V_{L}(t)$ at the load (result of the response to a ramped step) for the source-line-load example described in Section IV-B. Gray lines: MC samples (only 100 samples out of 50000 are shown); solid black line: mean $\mu_{V_{L}}$; dashed black line: 3-sigma deviations $\mu_{V_{L}} \pm 3 \sigma_{V_{L}}$.

\section{CONCLUSION}

The influence of the etching process on the behavior of an on-chip interconnect, more specifically of an IEM line, was

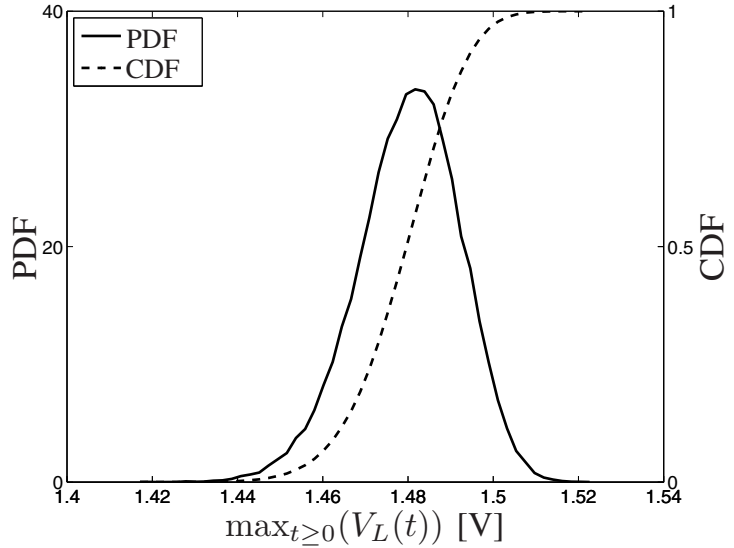

Fig. 8: PDF and $\mathrm{CDF}$ of the overshoot, i.e. the maximum value of $V_{L}(t)$ as presented in Fig. 7.

studied. To this end, the p.u.l. parameters of the line were macromodeled starting from a limited number of computationally expensive but accurate EM simulations. The macromodel describes the behavior of the interconnect not only as a function of frequency, but also as a function of a geometrical parameter that determines the shape of the cross-section of the IEM line. A variability analysis is carried out via MC runs in both the frequency and the time domain, allowing to assess the influence of this shape, and thus of the etching. It is shown that thanks to the macromodeling step, this approach is efficient, robust, and reliable.

\section{ACKNOWLEDGMENT}

This work was supported by the Fund for Scientific Research Flanders (FWO-Vlaanderen). Dirk Deschrijver is a post-doctoral research fellow of FWO-Vlaanderen.

\section{REFERENCES}

[1] W. Winkler, J. Borngräber, F. Korndörfer, and C. Scheytt, "94 GHz amplifier in SiGe technology," in Proceedings of the 38th European Microwave Conference, Amsterdam, the Netherlands, 2008, pp. 167-170.

[2] T. Demeester and D. De Zutter, "Quasi-TM transmission line parameters of coupled lossy lines based on the Dirichlet to Neumann boundary operator," IEEE Trans. Microw. Theory Tech., vol. 56, no. 7, pp. 16491660, Jul. 2008.

[3] T. Demeester, D. Vande Ginste, and D. De Zutter, "Accurate study of the electromagnetic and circuit behavior of finite conducting wedges and interconnects with arbitrary cross-sections," in Proc. of 19th IEEE Int. Conf. on Electrical Performance of Electronic Packaging and Systems (EPEPS), Austin, TX, USA, 24 - 27 Oct. 2010, pp. 133-136.

[4] D. Deschrijver and T. Dhaene, "Stability and passivity enforcement of parametric macromodels in time and frequency domain," IEEE Trans. Microw. Theory Tech., vol. 56, no. 11, pp. 2435-2441, Nov. 2008.

[5] G. Antonini, D. Deschrijver, and T. Dhaene, "Broadband rational macromodeling based on the adaptive frequency sampling algorithm and the partial element equivalent circuit method," IEEE Trans. Electromagn. Compat., vol. 50, no. 1, pp. 128-137, Feb. 2008.

[6] Q. Zhang, J. J. Liou, J. McMacken, J. Thomson, and P. Layman, "Development of robust interconnect model based on design of experiments and multiobjective optimization," IEEE Trans. Electron Devices, vol. 48, no. 9, pp. 1885-1891, Sep. 2001.

[7] B. Gustavsen and A. Semlyen, "Rational approximation of frequency domain responses by vector fitting," IEEE Trans. Power Del., vol. 14, no. 3, pp. 1052-1061, Jul. 1999.

[8] J. P. Berrut and L. N. Trefethen, "Barycentric lagrange interpolation," SIAM Review, vol. 46, no. 3, pp. 501-517, Sep. 2004. 